\title{
Síndrome de burnout en estudiantes de medicina: frecuencia, características y factores asociados
}

\section{Burnout syndrome in medical students: frequency, characteristics and associated factors}

\section{Correspondencia}

Jennifer Steffany Vilchez Cornejo

steffany578@gmail.com

Recibido: 13/11/2016

Arbitrado por pares

Aprobado: 21/12/2016

Citar como: Vilchez-Cornejo J, Huamán-Gutiérrez R, ArceVillalobos L, Morán-Mariños C Mihashiro-Maguiña K, Melo-Mallma $N$, et al. Síndrome de Burnout en estudiantes de medicina: frecuencia, características y factores asociados. Acta Med Peru. 2016;33(4):282-8
Jennifer Vilchez-Cornejo1,a, Roberto Huamán-Gutiérrez²,a, Laura Arce-Villalobos,a, Cristian Morán-Mariños 4,a, Kenyi Mihashiro-Maguiña5,a Niel Melo-Mallmab,a, Jorge Eyzaguirre-Villagarcia ${ }^{7, a}$, Carlos Rojas-Valle ${ }^{1}$

1 Facultad de Medicina Humana. Universidad Nacional de Ucayali. Ucayali, Perú.

2 Sociedad Científica de Estudiantes de Medicina de Ica (SOCEMI), Universidad Nacional San Luis Gonzaga de Ica. Ica, Perú.

3 Sociedad Científica de Estudiantes de Medicina Veritas (SCIEMVE), Universidad San Martin de Porres. Lima, Perú.

4 Sociedad Científica de Estudiantes de Medicina, Universidad Privada San Juan Bautista (SOCIEM UPSJB). Lima, Perú.

5 Facultad de Medicina Humana, Universidad Nacional José Faustino Sánchez Carrión. Lima, Perú.

6 Sociedad Científica de Estudiantes de Medicina del Centro (SOCIMEC), Universidad Nacional del Centro del Perú. Junín, Perú.

7 Escuela de Medicina Humana, Universidad Cesar Vallejo. Piura, Perú.

a Estudiante de Medicina

\section{RESUMEN}

Objetivo: Determinar la frecuencia, características y los factores que están asociados ante el síndrome de burnout (SB) en estudiantes de medicina. Materiales y métodos: Estudio transversal analítico. Se encuestaron a 583 estudiantes de medicina que cursaban las ciencias clínicas y realizaban rotaciones hospitalarias en los departamentos de Lima, Ica, Junín, Lambayeque y Ucayali. EI SB se evaluó a través del Maslach Burnout Inventory en su versión validada en español así mismo las características sociodemográficas que fueron incluidas en el instrumento Resultados: Se encontró que 50,6\% eran varones, el $16,5 \%, 47,7 \%$ y $35,3 \%$ de los estudiantes indicaron tener agotamiento emocional, despersonalización severa y realización personal baja, respectivamente. Se halló asociación significativa entre tener SB y sobrepeso (razón de prevalencias $[R P]=1,57$; intervalo de confianza al $95 \%[I C 95 \%]=1,14-2,14$ ), ser obeso $(R P=2,68 ; I C 95 \%=1,75-4,11)$, padecer una enfermedad crónica $(R P=1,84 ; I C 95 \%=1,38-2,45)$, fumar ( $R P=1,41 ; I C 95 \%=1,76-2,28)$ ingerir bebidas alcohólicas $(R P=1,46 ; I C 95 \%=1,10-1,95)$ y proceder de una universidad de provincia $(R P=1,20 ; \mid C 95 \%=1,05-1,36)$. Conclusión: EI SB se presentó en aquellos quienes padecían de alguna enfermedad crónica, fumaban, ingerían bebidas alcohólicas y procedían de una universidad de provincia.

Palabras clave: Agotamiento profesional; Estudiantes de medicina; Bebidas alcohólicas; Bebidas energéticas; Enfermedad crónica (fuente: DECS/Bireme). 


\begin{abstract}
Objective: To determine the frequency, characteristics and associated factors with the Burnout Syndrome (BS) in medical students. Material and methods: This is a cross-sectional study. Five-hundred and eighty three (583) medical students doing clinical sciences courses having hospital training periods in Lima, Ica, Junín, Lambayeque and Ucayali were surveyed. The presence of BS was determined using the Maslach Burnout Inventory (MBI) in its validated Spanish version, and also the socio-demographic characteristics included in the instrument. Results: Gender distribution was similar (50.6\% were male), also $16.5 \%, 47.7 \%$ and $35.3 \%$ of the students reported having emotional exhaustion, severe depersonalization and low personal accomplishments, respectively, as defined by the MBI. Also, a significant association was found between $\mathrm{BS}$ and being overweight (Prevalence ratio [PR] $=1.57 ; 95 \%$ confidence interval $[95 \% \mathrm{Cl}]=$ 1.14 to 2.14 ); obese ( $P R=2.68 ; 95 \% \mathrm{Cl}=1.75$ to 4.11 ), having chronic disease ( $P R=1.84 ; 95 \% \mathrm{Cl}=1.38$ to 2.45 ), smoking $(P R=1.41 ; 95 \% \mathrm{Cl}=1.76$ to 2.28$)$ drinking alcohol $(P R=1.46 ; 95 \% \mathrm{Cl}=1.10$ to 1.95$)$ and studying in a province university $(P R=1.20 ; 95 \% \mathrm{Cl}=1.05$ to 1.36). Conclusion: BS occurs in those who suffer from a chronic illness, smoke, drink alcohol, and study in a province university.
\end{abstract}

Keywords: Burnout, professional; Students, medical; Alcoholic beverages; Energy drinks; Chronic disease (source: $\mathrm{MeSH} / \mathrm{NLM})$.

\section{INTRODUCCIÓN}

Los elevados niveles de estrés y depresión en estudiantes de medicina tienen implicaciones considerables que van mucho más allá de la educación médica. Una revisión menciona prevalencias cercanas o superiores al 50\% de personas que tienen agotamiento emocional y despersonalización, llegándose a considerar ya como una epidemia ${ }^{[1]}$. En la actualidad, el conjunto de síntomas que se relacionan con el desgaste laboral y la asociación con las patologías descritas anteriormente se conoce como síndrome de burnout (SB) ${ }^{[2]}$.

Durante el año de 1981 se plantearon dos formas de definir conceptualmente al SB, una que lo definía en base a un constructo tridimensional: agotamiento emocional, despersonalización y baja realización personal; y la otra que lo hacía a través un constructo unidimensional que solo consideraba al agotamiento emocional [3]. Esta forma de conceptualizar al SB permitió observar que la escala de agotamiento emocional es la que se ajusta mejor a las variables relacionadas con el trabajo y el estudio. Por tanto, el contenido central del agotamiento profesional tendría que ver con una pérdida de las fuentes de energía del sujeto, una combinación de fatiga física, cansancio emocional y cognitivo ${ }^{[4-6]}$.

El SB, también conocido como síndrome de desgaste profesional, síndrome de sobrecarga emocional, síndrome del quemado o síndrome de fatiga en el trabajo, fue declarado en el año 2000 por la Organización Mundial de la Salud como un factor de riesgo laboral debido a su capacidad para afectar la calidad de vida, salud mental e incluso poner en riesgo la vida de quien lo sufre ${ }^{[7,8]}$. No obstante, el problema va más allá pues el que lo padece posiblemente brindará un servicio deficiente a los clientes, realizará sus funciones ineficientemente, tendrá un promedio de ausentismo mayor y expondrá a su centro laboral a pérdidas y fallos en el logro de objetivos ${ }^{[8,9]}$.
Padecer el SB durante la formación médica no es un evento benigno. Un estudio longitudinal realizado en siete escuelas de medicina en Estados Unidos evidencio una prevalencia del 49,6\% de $S B$, y el $11,2 \%$ de estos eran de 2 a 3 veces más propensos a presentar una ideación suicida, manteniéndose esta ideación durante un periodo de 12 meses en un 5.8\% en comparación de aquellos que no lo presentaban ${ }^{[10]}$.

Por otro lado, se sabe que aquellos estudiantes que obtenían puntajes altos en pruebas que tamizan depresión también mostraban una baja empatía con los pacientes ${ }^{[11]}$; es decir, el SB en los estudiantes de medicina se relacionaría con problemas de salud mental que a su vez ocasionarían efectos negativos en la atención al paciente, una baja identificación con la institución, fracaso académico e incluso intentos de suicidio ${ }^{[12]}$.

Cifras similares fueron encontradas en estudios latinoamericanos ${ }^{[13]}$. En el Perú, un estudio realizado en estudiantes de medicina encontró que el $57,6 \%$ de los entrevistados tenía al menos un componente alterado del instrumento Maslach Burnout Inventory (MBI) utilizado para identificar SB; además, el 24,1\% presentaba un nivel severo de agotamiento mientras otros presentaron un nivel severo de despersonalización $(45,6 \%)$, concluyendo que existía una alta frecuencia de casos de SB en esta población ${ }^{[14]}$.

La relevancia del fenómeno burnout entre los estudiantes universitarios y la detección precoz de niveles sintomáticos significativos puede constituir un indicador de posibles dificultades futuras, en los planos de éxito académico o profesional y una oportunidad excelente de intervención temprana ${ }^{[12,13]}$. Por lo tanto, el presente artículo busca hallar la frecuencia, características y factores asociados del agotamiento profesional en los estudiantes de medicina humana, estableciendo nueva información y más específica respecto a este fenómeno. 


\section{MATERIALES Y MÉTODOS}

\section{Diseño y población de estudio}

Se realizó un estudio analítico de tipo transversal, en el cual se aplicó una encuesta autoadministrada a estudiantes de medicina para determinar la frecuencia, características y factores asociados al SB. Se realizó un muestreo no probabilístico tipo censal en la Universidad Nacional de Ucayali (Ucayali), Universidad Nacional San Luis Gonzaga de Ica (Ica), Universidad Nacional del Centro del Perú (Junín), Universidad Privada San Juan Bautista (Lima), Universidad Privada San Martín de Porres - filial norte (Lambayeque) y la Universidad Nacional José Faustino Sánchez Carrión (Huacho); todas universidades, entre públicas y privadas, de cinco departamentos del Perú.

Se incluyó a todos los estudiantes matriculados del tercer al sexto año que cursan las ciencias clínicas y que realizaban rotaciones hospitalarias durante los meses correspondientes a junio del 2014 hasta agosto del 2015. Se excluyó a aquellos que no dieron su consentimiento para participar o en los que las encuestas fueran ilegibles $\mathrm{u}$ otras causas que imposibilitaran el análisis de los datos registrados.

\section{Variables y procedimiento}

Se aplicó una encuesta autoadministrada para la recolección de los datos, la cual medía la frecuencia, características y factores asociados al SB; la cual fue adaptada para la población de este estudio. Las variables evaluadas fueron edad, sexo, cantidad de horas de estudio, año de estudios, si desaprobó el ciclo anterior concluido a la fecha de la entrevista, índice de masa corporal (IMC) y hábitos nocivos (fumar, consumo de bebidas alcohólicas, consumo de café y consumo de bebidas energizantes). Para la detección del SB se hizo la evaluación en base a los siguientes casos: un nivel severo de agotamiento emocional; un nivel severo de despersonalización o un bajo nivel de realización personal ${ }^{[15]}$. Se consideró que una persona tenía SB si cumplía al menos uno de los tres criterios. No obstante, algunos autores recomiendan considerar la presencia de dos o más criterios para dar el diagnóstico de SB ${ }^{[14]}$. Por tal motivo, se hizo el análisis en ambas situaciones.

El instrumento se conformó por tres secciones. La primera sección recogía los datos personales de los estudiantes, tales como sexo, edad, universidad (publica/privada), ubicación de universidad (Lima/provincia), estado civil (soltero/casado/ conviviente), haber desaprobado algún curso, semestre académico en curso, horas de estudio a la semana, con quienes vivía y cómo solventaba sus gastos.

La segunda sección estaba dirigida para reconocer alguno de los factores que se encuentran asociados al SB, se consideraron el peso, la talla (ambos referidos por el entrevistado), además de las frecuencias en consumo de tabaco, alcohol, café, bebidas energéticas y antecedente de alguna enfermedad crónica.
La última sección correspondió al diagnóstico del SB a través del $\mathrm{MBI}$, cuestionario validado para población de habla hispana, que consta de 22 ítems ${ }^{[15]}$ los cuales miden tres componentes: agotamiento emocional (AE), despersonalización (DP) y realización personal (RP). Cada ítem cuenta con siete opciones de respuesta medidas en una escala tipo Likert que van de 0 (nunca) hasta 6 (todos los días). En base al puntaje obtenido se midieron los niveles de alteración que cuentan los componentes del SB, en donde los niveles de agotamiento emocional se evalúan en los ítems $1,2,3,6,8,13,14,16,20$ en el que se considera leve (0-18 puntos), moderado (19-26 puntos) y severo (27-54 puntos); niveles de despersonalización se evalúan en los ítems $5,10,11,15,22$ en el que considera leve (0-5 puntos), moderado (6-9 puntos) y severo (10-30 puntos) y los niveles de realización personal se evalúan en los ítems $4,7,9,12,17,18$, 19, 21 en donde se considera bajo (0-33 puntos), medio (34-39 puntos) y alto (40-48 puntos) ${ }^{[14]}$.

\section{Análisis de datos}

Los datos recogidos se registraron y clasificaron en una base elaborada en el programa Microsoft Excel 2010. Posteriormente, se hizo el análisis en STATA 11.0. Se presentaron los resultados de las variables cualitativas en frecuencias y porcentajes. Para las variables cuantitativas, se evaluó la normalidad a través de la prueba de Shapiro - Wilk $(p<0,05)$.

Para la estadística inferencial se usó la prueba de chi cuadrado para evaluar la asociación entre variables con más de dos categorías (padecer SB, horas semanales de estudio, etc.). Además, se realizó un análisis bivariado según sus características más importantes: padecer una enfermedad crónica, sobrepeso, fumar, ingerir bebidas alcohólicas y bebidas energéticas. Se hallaron razones de prevalencia (RP), un intervalo de confianza del $95 \%$ (IC95\%) y un valor de $p<0,05$ como estadísticamente significativo.

\section{Aspectos éticos}

El estudio fue aprobado por el Comité de Ética de la Facultad de Medicina Humana de la Universidad Nacional de Ucayali. Cada encuesta fue realizada previa firma del consentimiento informado. Se entregó una copia de esta al participante. En ella el encuestado podía solicitar el resultado después de haberlo rellenado (en el caso del diagnóstico de SB). Se omitió cualquier dato que permitiría su identificación.

\section{RESULTADOS}

La población total incluía a 640 estudiantes de medicina. De estos, 39 participantes no dieron su consentimiento (taza de rechazo del $6,1 \%$ ) y no fueron considerados dentro del estudio; posteriormente, se eliminaron 18 encuestas debido a que los registros eran ilegibles $u$ otras causas imposibilitaban su análisis $(2,8 \%)$. Al final, fueron incluidos en el estudio 583 estudiantes (Figura 1). En suma, el $8,9 \%$ de la población en general no fue incluida. 


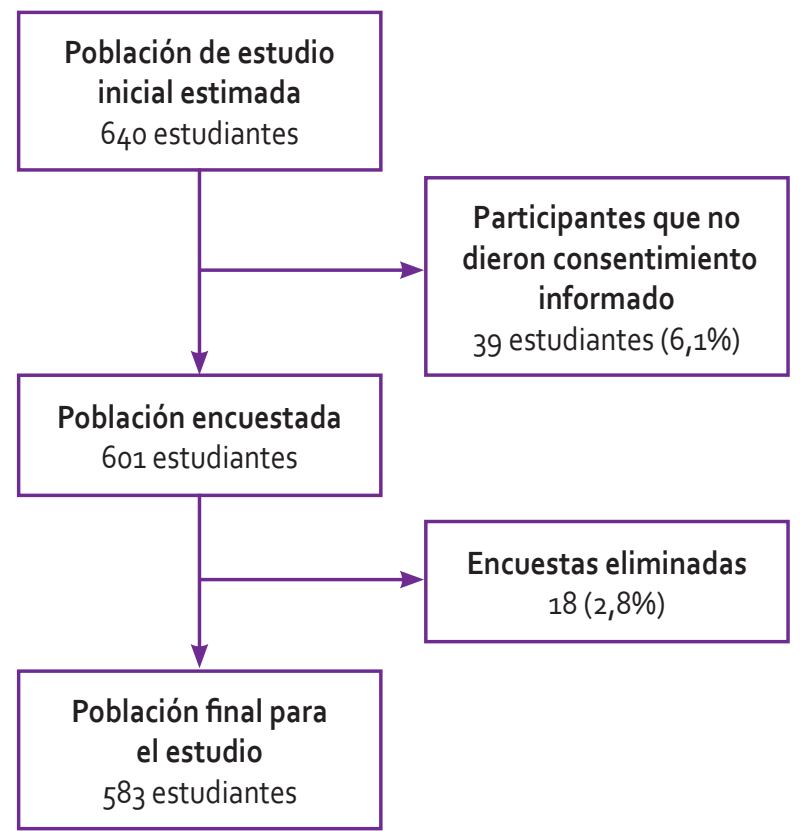

Figura 1. Flujograma de la población estudiada.

Tabla 1. Características generales de los estudiantes de medicina humana.

\begin{tabular}{|c|c|c|}
\hline Variable & $\mathbf{N}$ & $\%$ \\
\hline Edad & $23 \pm 3,40^{*}$ & $17-45^{\star *}$ \\
\hline Horas de estudio & $12,5 \pm 9,95^{\star}$ & $1-80^{\star \star}$ \\
\hline \multicolumn{3}{|l|}{ Sexo } \\
\hline Masculino & 295 & 50,6 \\
\hline Femenino & 288 & 49,4 \\
\hline \multicolumn{3}{|l|}{ Año de estudios } \\
\hline $3^{\mathrm{a}}$ año & 110 & 18,9 \\
\hline $4^{\mathrm{a}}$ año & 150 & 25,7 \\
\hline $5^{\mathrm{a}}$ año & 204 & 35 \\
\hline $6^{\mathrm{a}}$ año & 119 & 20,4 \\
\hline \multicolumn{3}{|c|}{ Desaprobó el Ciclo } \\
\hline \multicolumn{3}{|l|}{ Pasado } \\
\hline Si & 103 & 17,7 \\
\hline No & 480 & 82,3 \\
\hline IMC & $23,7 \pm 3,15^{\star}$ & $17-35^{\star *}$ \\
\hline \multicolumn{3}{|l|}{ Hábitos Nocivos } \\
\hline \multicolumn{3}{|l|}{ Fuma } \\
\hline Si & 92 & 15,8 \\
\hline No & 491 & 84,2 \\
\hline \multicolumn{3}{|c|}{ Bebidas alcohólicas } \\
\hline Si & 215 & 36,9 \\
\hline No & 368 & 63,1 \\
\hline \multicolumn{3}{|l|}{ Consumo de café } \\
\hline $\mathrm{Si}$ & 240 & 41,2 \\
\hline No & 343 & 58,8 \\
\hline \multicolumn{3}{|c|}{ Bebidas energizantes } \\
\hline $\mathrm{Si}$ & 121 & 20,8 \\
\hline No & 462 & 79,2 \\
\hline
\end{tabular}

Tabla 2. Características del síndrome de burnout en los estudiantes de medicina.

\begin{tabular}{lcc}
\multicolumn{1}{c}{ Variable } & N & $\%$ \\
\hline Agotamiento Emocional & & \\
Leve & 306 & 52,5 \\
Moderado & 181 & 31 \\
Severo & 96 & 16,5 \\
Despersonalización & & \\
Leve & 178 & 30,5 \\
Moderado & 127 & 21,8 \\
Severo & 278 & 47,7 \\
Realización Personal & & \\
Bajo & 206 & 35,3 \\
Medio & 128 & 22 \\
Alto & 249 & 42,7 \\
Burnout (Con dos o más componentes) & \\
Si & 141 & 24,1 \\
No & 442 & 75,8 \\
\hline
\end{tabular}

El 50,6\% (295) de los entrevistados fueron varones, la edad promedio fue de $23 \pm 3,4$ años. Respecto al año de estudios el $35 \%$ (204) cursaba el quinto año y el promedio de horas de estudio por semana fue de $12,5 \pm 9,95$ horas. El IMC promedio fue de $23,7 \mathrm{~kg} / \mathrm{m}^{2}$ (rango: 17 a $35 \mathrm{~kg} / \mathrm{m}^{2}$ ). Por otro lado, respecto a los hábitos nocivos, el consumo de café predominó con 41,2\% (240), mientras que sólo el 15,8\% (92) fumaba cigarrillos (Tabla 1).

La Tabla 2 muestra las características del SB en los encuestados según los tres componentes del cuestionario MBI. El 16,5\% (96) de los estudiantes tuvieron AE severo, el 47,7\% (278) presentó una DP severa mientras que el $42,7 \%$ (249) tuvo RP alto. De manera global el 24,2\% (141) padecía SB diagnosticado con dos o más componentes del MBI.

El 37,4\% tenía el antecedente de tener una enfermedad crónica; de la misma forma respecto a fumar $(38,0 \%)$, consumo de bebidas alcohólicas $(30,2 \%)$, consumo de café $(25,0 \%)$, consumo de bebidas energizantes $(20,7 \%)$ y sobrepeso $(35,1 \%)$. Se encontró una asociación significativa entre estos factores y la presencia de SB. En relación con las horas de estudio, el 56\% de los estudiantes con SB estudiaban menos de 10 horas a la semana $(p<0,01)$.

En relación al diagnóstico de $\mathrm{SB}$ con al menos un componente alterado, todas las edades tuvieron una asociación negativa: $20-$ 25 años (RP: 0,78; IC: 0,68-0,88), 26 - 30 años (RP: 0,70; IC: 0,55 -0,89) y más de 30 años (RP: 0,70; IC: 0,49-0,99) en relación con ser menor de 20 años. El recibir dinero de sus padres y trabajar (RP: 0,75 ; IC: 0,60-0,92) también disminuía la probabilidad de padecer SB en comparación de aquellos que sólo reciben dinero de sus padres. El cursar el sexto año de medicina (RP: 0,$74 ;$ IC: $0,62-0,88$ ) presentaba un $26 \%$ menos probabilidad de padecer SB en comparación de los que cursaban el tercer año. 
Tabla 3. Análisis bivariado del síndrome de burnout y sus factores asociados.

\begin{tabular}{|c|c|c|c|c|}
\hline \multirow{2}{*}{ Variables } & \multicolumn{2}{|c|}{$\begin{array}{l}\text { Síndrome de burnout } \\
\text { (uno o más componentes) }\end{array}$} & \multicolumn{2}{|c|}{$\begin{array}{c}\text { Síndrome de burnout } \\
\text { (dos o más componentes) }\end{array}$} \\
\hline & RP & IC95\% & $\mathbf{R P}$ & IC95\% \\
\hline \multicolumn{5}{|l|}{ Sexo } \\
\hline Femenino & Ref. & & Ref. & \\
\hline Masculino & 1,02 & $0,92-1,15$ & 1,11 & $0,83-1,48$ \\
\hline \multicolumn{5}{|l|}{ Edad } \\
\hline$<20$ años & Ref. & & Ref. & \\
\hline 20 - 25 años & 0,78 & $0,68-0,88$ & 1,37 & $0,72-2,64$ \\
\hline 26 - 30 años & 0,70 & $0,55-0,89$ & 1,70 & $0,80-3,62$ \\
\hline Mayor de 30 & 0,70 & $0,49-0,99$ & 1,75 & $0,72-4,23$ \\
\hline \multicolumn{5}{|l|}{ Estado Civil } \\
\hline Soltero & Ref. & & Ref. & \\
\hline Casado & 0,95 & $0,64-1,41$ & 1,82 & $0,98-3,41$ \\
\hline Conviviente & 1,48 & $1,40-1,57$ & 1,82 & $0,76-4,35$ \\
\hline \multicolumn{5}{|l|}{ Universidades } \\
\hline Lima & Ref. & & Ref. & \\
\hline Provincia & 1,20 & $1,05-1,36$ & 0,60 & $0,45-0,79$ \\
\hline \multicolumn{5}{|l|}{ Índice de masa corporal } \\
\hline$<25 \mathrm{~kg} / \mathrm{m}^{2}$ & Ref. & & Ref. & \\
\hline $25-30 \mathrm{~kg} / \mathrm{m}^{2}$ & 1,00 & $0,87-1,14$ & 1,57 & $1,14-2,14$ \\
\hline$>30 \mathrm{~kg} / \mathrm{m}^{2}$ & 1,07 & $0,82-1,40$ & 2,68 & $1,75-4,11$ \\
\hline Enfermedad crónica * & 1,07 & $0,95-1,22$ & 1,84 & $1,38-2,45$ \\
\hline Fuma * & 1,19 & $1,05-1,34$ & 1,41 & $1,76-2,28$ \\
\hline Alcohol * & 1,00 & $0,89-1,12$ & 1,46 & $1,10-1,95$ \\
\hline Consumo de café * & 1,12 & $1,01-1,25$ & 1,05 & $0,79-1,42$ \\
\hline Bebidas energéticas * & 1,06 & $0,93-1,21$ & 0,82 & $0,56-1,21$ \\
\hline \multicolumn{5}{|l|}{ Solventa sus gastos } \\
\hline Recibe dinero de padres & Ref. & & Ref. & \\
\hline Trabaja & 0,87 & $0,68-1,11$ & 1,01 & $0,57-1,78$ \\
\hline Recibe dinero de padres y trabaja & 0,75 & $0,60-0,92$ & 1,21 & $0,82-1,77$ \\
\hline \multicolumn{5}{|l|}{ Año académico } \\
\hline 3er año & Ref. & & Ref. & \\
\hline 4to año & 0,71 & $0,60-0,83$ & 1,15 & $0,72-1,82$ \\
\hline 5to año & 0,88 & $0,78-0,99$ & 1,29 & $0,84-1,98$ \\
\hline 6 to año & 0,74 & $0,62-0,88$ & 1,08 & $0,66-1,77$ \\
\hline
\end{tabular}

* Referencia correspondió a la ausencia de la variable estudiada. RP: razón de prevalencias; IC95\%: intervalo de confianza al $95 \%$.

También se encontró que el provenir de una universidad de provincia (RP: 1,20 ; IC: $1,05-1,36$ ) se asoció positivamente, incrementando en 1,20 veces la probabilidad de padecer SB en comparación a los provenientes de una universidad limeña. Asimismo, fumar (RP: 1,19; IC: 1,05-1,34) y consumir café (RP: $1,12 ; \mid C: 1,01-1,25)$ se asociaron positivamente, incrementando en 1,19 y 1,12 veces, respectivamente la probabilidad de padecer SB (Tabla 3).

Finalmente, en la misma tabla, usando el diagnóstico de SB con al menos dos o más componentes alterados, se observó que el provenir de una universidad de provincia (RP: 0,60; IC: $0,45-0,79)$ se asoció negativamente. En contraste el presentar IMC dentro del intervalo de $25-30 \mathrm{~kg} / \mathrm{m}^{2}$ (RP: 1,57 ; IC: $1,14-2,14)$ y $>30 \mathrm{~kg} / \mathrm{m}^{2}$ (RP: 2,68 ; IC: $\left.1,75-4,11\right) \mathrm{se}$ asociaron positivamente, incrementando en 1,57 y 2,68 veces respectivamente la probabilidad de padecer SB comparado con los que tenían IMC $<25 \mathrm{~kg} / \mathrm{m}^{2}$. También el fumar (RP: 1,41 ; IC: $1,76-2,28$ ), consumir bebidas alcohólicas (RP: 1,46; IC: $1,10-1,95)$ y tener una enfermedad crónica (RP: 1,84 ; IC: $1,38-2,45)$ se asociaron positivamente, e incrementaron la probabilidad de padecer SB. 


\section{DISCUSIÓN}

Durante el presente estudio se pudo observar que por lo menos dos de cada diez estudiantes de medicina presentaron SB. Estudios similares realizados en Chile, Colombia y Brasil ${ }^{[16-18]}$ nos muestran que existe poca frecuencia para desarrollarse este agotamiento profesional, sin embargo a pesar de que se presente muy pocas veces, aquellos estudiantes que lo padecen están predispuestos a presentar mayores problemas de salud mental ocasionando una problemas para identificarse con su institución, tener problemas académicos, e incluso que se presenten intentos de suicidio ${ }^{[2]}$, por lo que el manejo del SB también debe contemplar los factores asociados.

Se encontró que, aquellos estudiantes que tuvieron sobrepeso y obesidad estaban asociados a padecer SB a diferencia de los que contaban con un IMC normal. Esto podría explicarse debido a que el agotamiento profesional puede representar una respuesta de estrés crónico resultando nocivo para su entorno, una asociación que predispondría a una estimulación crónica del eje hipotálamo-hipófisis-suprarrenal y que a medio o largo plazo provocaría un aumento en la ingesta y peso corporal debido al exceso de liberación de glucocorticoides ${ }^{[19]}$.

El permanecer constantemente en un estado de estrés crónico y mental provoca concentraciones elevadas de interleucina 6 y proteína $C$ reactiva ${ }^{[20]}$ provocando en algunos isquemia, alteraciones de los niveles de glucemia, lipemia, presión arterial y frecuencia cardiaca alterada; y que incluso padecer una enfermedad crónica predispone en $84 \%$ la probabilidad de presentar un $\mathrm{SB}^{[22]}$; dichos hallazgos respaldarían la relación que se encontró en nuestro estudio.

Durante nuestro estudio se encontraron asociaciones significativas en aquellos que tenían hábitos de fumar, consumir bebidas alcohólicas y consumir bebidas energizantes. Dichos hallazgos son similares a los encontrados en un estudio realizado en Colombia ${ }^{[17]}$. El afrontar constantemente los retos del pregrado motiva al estudiante a utilizar diferentes métodos para mejorar su rendimiento académico y vencer el sueño ${ }^{[22]}$. Aquel que fuma debido a un severo agotamiento profesional lo haría como mecanismo para librarse del estrés crónico, ya que la nicotina puede actuar como un estimulante, en situaciones de fatiga, pero a la vez como tranquilizantes cuando existen niveles elevados de ansiedad y estrés ${ }^{[23]}$, llevando a aquellos fumadores a realizar estas prácticas en cualquier situación predisponiendo y manteniendo el estado de estrés y agostamiento crónico.

No existen muchos reportes y estudios realizados en estudiantes con SB que se encuentren realizando prácticas en el área clínica; sin embargo, Santen ${ }^{[24]}$ en un estudio realizado en estudiantes de medicina mostró la asociación entre el SB y el año de estudios, así el primer año tuvo una frecuencia de SB del $21 \%$, segundo $41 \%$, tercero $43 \%$ y cuarto de $31 \%$; a pesar de no medir los mismos años, salvo el cuarto, la frecuencia en nuestro estudio fue menor. En el mismo estudio, el poco apoyo, los niveles altos de estrés y la falta de control sobre su propia vida estarían directamente relacionados con la presencia de SB. Sería adecuado investigar por qué en nuestro caso los años mayores tuvieron menos probabilidad de presentar SB a pesar que supondría que a mayor grado, habría mayor responsabilidad.

Las limitaciones encontradas en nuestro estudio correspondieron al sesgo de selección al no realizar una muestra aleatorizada y que se incluyó únicamente a los estudiantes de ciencias clínicas y no a aquellos estudiantes de ciencias básicas, por lo que los resultados no serían extrapolables. Uno de nuestros fines fue captar a los estudiantes que aparentemente estaban más predispuestos a tener un SB. Entendimos que los estudiantes de últimos años tendrían mayor carga académica lo que lo haría más proclives a desarrollar la enfermedad estudiada. Además, el estudio evalúo solo algunas de las características académicas y hábitos de la población. Sin embargo, este estudio nos permite reconocer asociaciones importantes entre los estudiantes de medicina que deberán ser evaluados en estudios posteriores.

Recomendamos el desarrollo de un estudio aleatorizado que tenga en consideración la salud mental y los niveles de dependencia de los hábitos nocivos (alcoholismo, consumo de cigarrillos o energizantes) para que se puedan tomar medidas preventivas y de afronte que mejoren la calidad de vida y el desempeño académico de los estudiantes. Se concluye que los estudiantes de medicina que tienen mayor predisposición a padecer agotamiento profesional son aquellos quienes tienen una enfermedad crónica, fuman, ingieren bebidas alcohólicas y proceden de una universidad de provincia.

Fuente de financiamiento: El presente estudio ha sido autofinanciado por los autores.

Declaración de conflictos de intereses: Los autores declaran no tener conflictos de interés con respecto a la publicación del presente estudio.

\section{REFERENCIAS BIBLIOGRÁFICAS}

1. West CP, Dyrbye LN, Erwin PJ, Shanafelt TD. Interventions to prevent and reduce physician burnout: a systematic review and meta-analysis. Lancet. 2016;388(10057):2272-81.

2. Arango-Agudelo $\mathrm{S}$, Castaño-Castrillón JJ, Henao-Restrepo CJ, Jiménez-Aguilar DP, López-Henao AF, Páez-Cala ML. Síndrome de Burnout y factores asociados en estudiantes de I a X semestre de la facultad de medicina de La Universidad de Manizales (Colombia), 2009. Arch Med (Manizales). 2010;10(2):110-26.

3. Maslach C, Jackson SE. The measurement of experienced burnout. J Organiz Behav. 1981;2(2):99-113.

4. Sandra-Carlotto M, Gonçalves-Câmara S, Brazil-Borges AM Predictores del sindrome de burnout en estudiantes de un curso técnico de enfermería. Diversitas. 2005;1(2):195-204.

5. Gutiérrez-Rico D. El Síndrome de Burnout en alumnos de educación secundaria. INED. 2008;5(10):26-35.

6. Rosales-RicardoY. Estudio unidimensional del síndrome de burnout en estudiantes de medicina de Holguín. Rev Asoc Esp Neuropsiq. 2012;32(116):795-803.

7. Gutiérrez-Aceves GA, Celis-López MA, Moreno-Jiménez $S$, Farias-Serratos F, Suárez-Campos JJ. Síndrome de burnout. Arch Neurocien (Mex). 2006;11(4):305-9.

8. Saborío-Morales L, Hidalgo-Murillo LF. Síndrome de Burnout. Med Leg Costa Rica. 2015;32(1):119-24. 
9. Rodríguez-Ramírez J. Propuesta: Programa Integral de Autocuidado dirigido a personal de enfermería. Enfermería en Costa Rica. 2010;31(1):2-7.

10. Dyrbye L, Thomas M, Massie S, Power D, Eacker A, HarperW, et al. Burnout and suicidal ideation among U.S. medical students. Ann Intern Med. 2008;149(5):334-41.

11. Maslach C, Shaufeli W, Leiter M. Job burnout. Annu Rev Psychol. 2001;52:397-422.

12. Estela-Villa LM, Jiménez-Román CR, Landeo-Gutiérrez JS, Tomateo-Torvisco JD, Vega-Dienstmaier JM. Prevalencia de síndrome de Burnout en alumnos del séptimo año de medicina de una universidad privada de Lima, Perú. Rev Neuropsiquiatr Perú. 2010;73(4):147.

13. Reyes-Ticas A, Medina MT, MesaX, ParedesY, BarahonaY, Sierra M. Estudio de síndrome de "Burnout", depresión y factores asociados en los practicantes internos del Hospital Escuela. Rev Fac Cienc Méd. 2012;9(1)14-20.

14. Nakandakari MD, De la Rosa DN, Failoc-Rojas VE, Huahuachampi $\mathrm{KB}$, Nieto WC, Gutiérrez Al, et al. Síndrome de burnout en estudiantes de medicina pertenecientes a sociedades científicas peruanas: Un estudio multicéntrico. Rev Neuropsiquiatr. 2015;78(4):203-10.

15. Vásquez-Manrique JF, Maruy-Saito A, Verne-Martín E. Frecuencia del síndrome de burnout y niveles de sus dimensiones en el personal de salud del Servicio de Emergencia de Pediatría del Hospital Nacional Cayetano Heredia en el año 2014. Lima, Perú. Rev Neuropsiquiatr. 2014;77(3):168-74.
16. Alfaro-Toloza P, Olmos-de-Aguilera R, Fuentealba M, CéspedesGonzález E. Síndrome de Burnout y factores asociados en estudiantes de una escuela de medicina de Chile. CIMEL. 2013;18(2):23-6.

17. Serrano FT, Salguero-Sánchez J, Ayala-Fernández J, García-Torres MF, Meza JC, Mejía CR. Síndrome de Burnout en estudiantes de seis facultades de medicina de Colombia, 2016-1: estudio multicéntrico. CIMEL. 2016;21(2):29-34.

18. De Cavalcante $G$, Ribeiro $H$, De Almeida PC, De Cavalcante B, Holanda $G$. The prevalence of burnout syndrome in medical students. Arch Clin Psychiatry (São Paulo). 2016;43(1):6-10.

19. Adam TC, Epel ES. Stress, eating and the reward system. Physiol Behav. 2007;91(4):449-58.

20. Ranjit N, Diez-RouxAV, Shea S, Cushman M. Psychosocial factors and inflammation in the multi-ethnic study of atherosclerosis. Arch Intern Med. 2007;167(2):174-83.

21. Ranchal-Sánchez A, Vaquero-Abellán M. Burnout, variables fisiológicas y antropométricas: un estudio en el profesorado. Med Segur Trab. 2008;54(210):47-55

22. Manrique FG, Ospina-Díaz JM, Barrera LF. Prevalencia de consumo de bebidas energizantes en estudiantes del área de la salud. Tunja, Boyacá, 2014. Rev Salud Hist Sanid. 2015;10(1):3-13.

23. Becoña E. Tabaco, ansiedad y estrés. Salud Drog. 2003;3(1):70-92.

24. Santen SA, Holt DB, Kemp JD, Hemphill RR. Burnout in medical students: examining the prevalence and associated factors. South Med J. 2010;103(8):758-63.

\section{Las ediciones anteriores de Acta Médica Peruana están disponibles en:}

\section{www.scielo.org.pe}

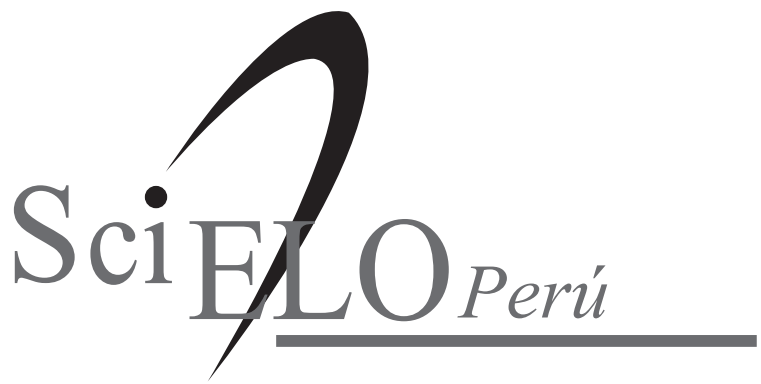

